\title{
ASPECTOS BIOLÓGICOS DE Halysidota pearsoni (LEPIDOPTERA: ARCTIIDAE) COM FOLHAS DE AMOREIRA ${ }^{1}$
}

\author{
Fabricio Fagundes Pereira², José Cola Zanuncio², Olaf Hermann Hendrik Mielke ${ }^{3}$, Juliana Maria de \\ Oliveira $^{2}$, Carlos Eduardo Costa Paiva ${ }^{2}$ e Glauco da Cruz Canevari ${ }^{4}$
}

\begin{abstract}
RESUMO - O objetivo deste trabalho foi estudar aspectos biológicos de Halysidota pearsoni Watson, 1980 (Lepidoptera: Arctiidae). Lagartas de H. pearsoni foram criadas com folhas de Morus alba L. em potes plásticos até a fase de pupa. Dez casais desse lepidóptero foram individualizados em gaiolas para obtenção de ovos à temperatura de $25 \pm 2{ }^{\circ} \mathrm{C}$, umidade relativa de $60 \pm 10 \%$ e fotofase de 12 horas. Halysidota pearsoni teve período de oviposição de $3,5 \pm 0,17$ com $141,00 \pm 9,18$ ovos por fêmea, período de incubação de 7,5 $\pm 0,17$ dias e viabilidade de ovos de 53,34 $\pm 5,24 \%$. A fase larval de $H$. pearsoni teve seis estádios, com duração total de 28 dias, viabilidade de $91,78 \pm 3,24 \%$. A duração e a viabilidade dos períodos pré-pupal e pupal de $H$. pearsoni foram, respectivamente, de 7,0 \pm 00 e 19,39 \pm 0,74 dias e de 70,15 $\pm 5,63$ e 93,62 $\pm 3,60 \%$. O peso médio de suas pupas foi de $464,17 \pm 7,70 \mathrm{mg}$ e a razão sexual e de 0,45 . A longevidade (dias) de machos

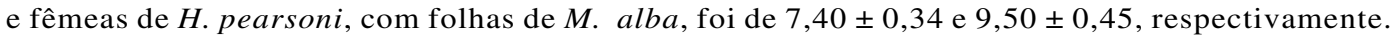

Palavras chave: Insecta, biologia e Morus alba.

\section{BIOLOGICAL ASPECTS OF Halysidota pearsoni (LEPIDOPTERA: ARCTIIDAE) FED WITH LEAVES OF Morus alba}

\begin{abstract}
The objective of this work was to study biological aspects of Halysidota pearsoni Watson, 1980 (Lepidoptera: Arctiidae). Caterpillars of $\boldsymbol{H}$. pearsoni were fed with leaves of Morus alba L. in plastic pots until pupation. Ten pairs of this insect were individualized in cages for obtain eggs at the temperature of $25 \pm 2{ }^{\circ} \mathrm{C}$, relative humidity of $60 \pm 10 \%$ and photo phase of 12 hours. Halysidota pearsoni had an oviposition period of $3.5 \pm 0.17$ with $141.00 \pm 9.18$ eggs perfemale; an incubation period of $7.5 \pm 0.17$ days and an egg viability of $53.34 \pm 5.24 \%$. The larva stage of $\boldsymbol{H}$. pearsoni had six instars with a total duration of 28 days and viability of $91.78 \pm 3.24 \%$. The duration and the viability of the pre-pupa and pupa periods of $\boldsymbol{H}$. pearsoni was $7.0 \pm 0.0$ and $19.39 \pm 0.74$ days and $70.15 \pm 5.63$ and $93.62 \pm 3.60 \%$ respectively. The pupae mean weight was $464.17 \pm 7.70 \mathrm{mg}$ and sex rate 0.45 . The longevity (days) of males and females of $\boldsymbol{H}$. pearsoni was $7.40 \pm 0.34$ and $9.50 \pm 0.45$, respectively.
\end{abstract}

Keywords: Insecta, biology, Morus alba.

\footnotetext{
${ }^{1}$ Recebido em 28.04.2006 e aceito para publicação em 15.10.2006.

${ }^{2}$ Departamento de Biologia Animal/BIOAGRO, Universidade Federal de Viçosa, 36.570-000 Viçosa, Estado de Minas Gerais, Brasil.E-mail: <zanuncio@ufv.br>; <ffpereira@ insecta.ufv.br>; <juinsetos@yahoo.com.br>; <bigodecarlos@yahoo.com.br>. ${ }^{3}$ Departamento de Zoologia, Setor de Ciências Biológicas, Universidade Federal do Paraná, Caixa Postal 19020, 81531-980 CuritibaPR, Brasil.E-mail:<omhesp@ufpr.br>.

${ }^{4}$ Departamento de Fitotecnia da Universidade Federal de Viçosa - UFV.
} 


\section{INTRODUÇÃO}

O gênero Halysidota Hubner possui um complexo de aproximadamente 46 espécies, dos Estados Unidos até a Bolívia e Argentina, sendo algumas consideradas pragas secundárias em florestas e árvores ornamentais na América do Norte (WATSON e GOODGER, 1986). No Brasil, há relatos da coleta de Halysidota sp. em plantio de Eucalyptus sp. no Alto São Francisco, Minas Gerais (ZANUNCIO et al., 1989) e em fragmentos de floresta estacional residual em Itaara, Rio Grande do Sul (VIANA e COSTA, 2001).

Halysidota orientalis Rothschild, 1909 (Lepidoptera: Arctiidae) foi registrada em Morus alba L. em Piracicaba, São Paulo (SOTO-SANCHEZ et al., 2004) e Halysidota interlineata Walker, 1855 (Lepidoptera: Arctiidae) nessa planta no Estado do Rio de Janeiro (SILVA et al., 1968). Halysidota pearsoni Watson, 1980 (Lepidoptera: Arctiidae) foi registrada nos Estados do Rio Grande do Sul, Santa Catarina, Paraná, Minas Gerais, Rio de Janeiro, São Paulo, Goiás, Espírito Santo e de Pernambuco, no Brasil, e Villarica, no Paraguai, mas as plantas hospedeiras desse inseto não foram citadas (WATSON, 1980). Embora essas espécies tenham sido registradas em plantas de amoreira no Brasil, a única espécie de Lepidoptera citada como praga dessa cultura no país foi Automeris memusae (Walker, 1855) (Lepidoptera: Saturniidae) (FONSECA e FONSECA, 1986; GALLO et al., 2002).

O objetivo deste trabalho foi estudar aspectos biológicos de $H$. pearsoni a partir de lagartas dessa espécie coletadas em plantas de $M$. alba em Viçosa, Minas Gerais.

\section{MATERIAL E MÉTODOS}

Posturas de um lepidóptero foram coletadas em dezembro de 2004 em plantas de $M$. alba na área experimental do Laboratório de Entomologia Florestal da Universidade Federal de Viçosa (UFV), em Viçosa, Estado de Minas Gerais, com $20^{\circ} 45^{\prime}$ latitude sul, $42^{\circ}$ $51^{\prime}$ longitude oeste e altitude de $651 \mathrm{~m}$. Essas posturas foram acondicionadas em uma placa de Petri, de plástico, de $9,0 \mathrm{~cm}$ de diâmetro por $1,2 \mathrm{~cm}$ de altura, à temperatura de $25 \pm 2{ }^{\circ} \mathrm{C}$, umidade relativa de $60 \pm 10 \%$ e fotofase de $12 \mathrm{~h}$, com um chumaço de algodão embebido em água destilada. Noventa lagartas recém-eclodidas foram colocadas em grupos de cinco, por pote plástico de $10 \mathrm{~cm}$ de diâmetro por $10 \mathrm{~cm}$ de altura $(500 \mathrm{ml})$, com a tampa telada no centro. Um tubo de vidro, tipo anestésico, com água e folhas de $M$. alba, com os pecíolos envoltos por algodão úmido, foram colocadas no interior dos potes, e trocadas diariamente. Pupas desse lepidóptero foram colocadas em potes plásticos $(500 \mathrm{ml})$ e 10 espécimes adultos recém-emergidos foram montados, fotografados e enviados ao Departamento de Zoologia, Setor de Ciências Biológicas, da Universidade Federal do Paraná, em Curitiba, Estado do Paraná, Brasil, para identificação. Esses insetos foram identificados como $H$. pearsoni. Dez casais desse lepidóptero foram individualizados em gaiolas teladas de $30 \times 30 \times 30 \mathrm{~cm}$, com fundo de madeira e tampas de vidro com galhos de $M$. alba no seu interior, envoltos em chumaços de algodão para obtenção de ovos. O mesmo procedimento foi realizado na segunda geração para estudar a biologia de $H$. pearsoni. Foram observados o período embrionário e viabilidade de ovos, número de estádios, período e a viabilidade das fases de larva, pré-pupa e pupa, razão sexual, longevidade de adultos e número de posturas por fêmea de $H$. pearsoni. O número e a duração de cada estádio foram obtidos pela medição diária da cápsula cefálica das lagartas com microscópio estereoscópico e ocular graduada. Adultos dessa mariposa foram depositados no museu de entomologia da UFV, em Viçosa, Minas Gerais.

\section{RESULTADOS E DISCUSSÃO}

\subsection{Ovos}

Os períodos (dias) de oviposição, incubação, viabilidade dos ovos (\%) e número de ovos por fêmea de $H$. pearsoni foram de $3,50 \pm 0,17 ; 7,50 \pm 0,17 ; 53,34$ $\pm 5,24 ; 141,00 \pm 9,18$, respectivamente, para esse inseto criado com folhas de M. alba. Esses valores são próximos ao relatados para Idalus admirabilis (Cramer, 1777) (Lepidoptera: Arctiidae) com período de pré-oviposição de 3,3 $\pm 0,67$ dias, $157,9 \pm 0,67$ ovos por fêmea e viabilidade de ovos de $41,7 \%$, para esse inseto criado com folhas de Eucalyptus urophylla S.T. Blake (SANTOS et al., 2006). O número de ovos por fêmea de $H$. pearsoni nas primeira, segunda, terceira, quarta e quinta posturas foi de $68,00 \pm 11,72 ; 42,40 \pm 3,30 ; 26,75 \pm 4,26 ; 20,00$ $\pm 4,58 ; 13,50 \pm 3,50$, respectivamente. Seus ovos foram colocados em grupos ou isolados, nas laterais, na tela das gaiolas e na parte dorsal das folhas de amoreira. Ovos de $H$. pearsoni apresentaram coloração branca, tendendo à amarela próximo à eclosão. São de formato 
oval, com diâmetro aproximado de 1,01 mm (Figura 1A). Ovos dessa espécie assemelham-se aos de Eupseudosoma involuta (Sepp, [1849]) (Lepidoptera: Arctiidae), que apresentam coloração amarelo-esverdeada, forma semi-esférica com diâmetro de $0,98 \mathrm{~mm}$ e que, também, são colocados isolados ou em grupos na parte superior das folhas de eucalipto, confirmando
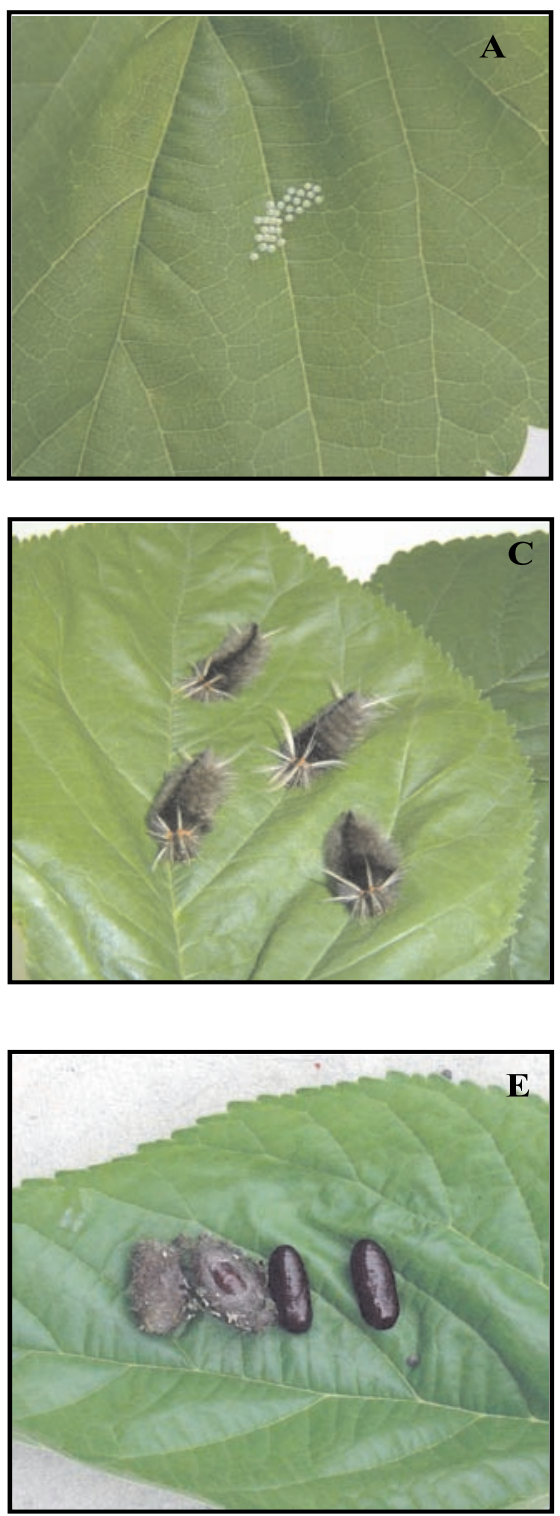

características morfológicas e comportamentais de espécies da família Arctiidae (ZANUNCIO, 1993). Ovos de Mallocephala deserticola (Berg, 1875) (Lepidoptera: Arctiidae), também, são semi-esféricos, com diâmetro de 1,07 mm, mas sua coloração de branco-amarelada se altera para cinza-escuro, quando próximos à eclosão (BENTANCOURT e SCATONI, 1998).
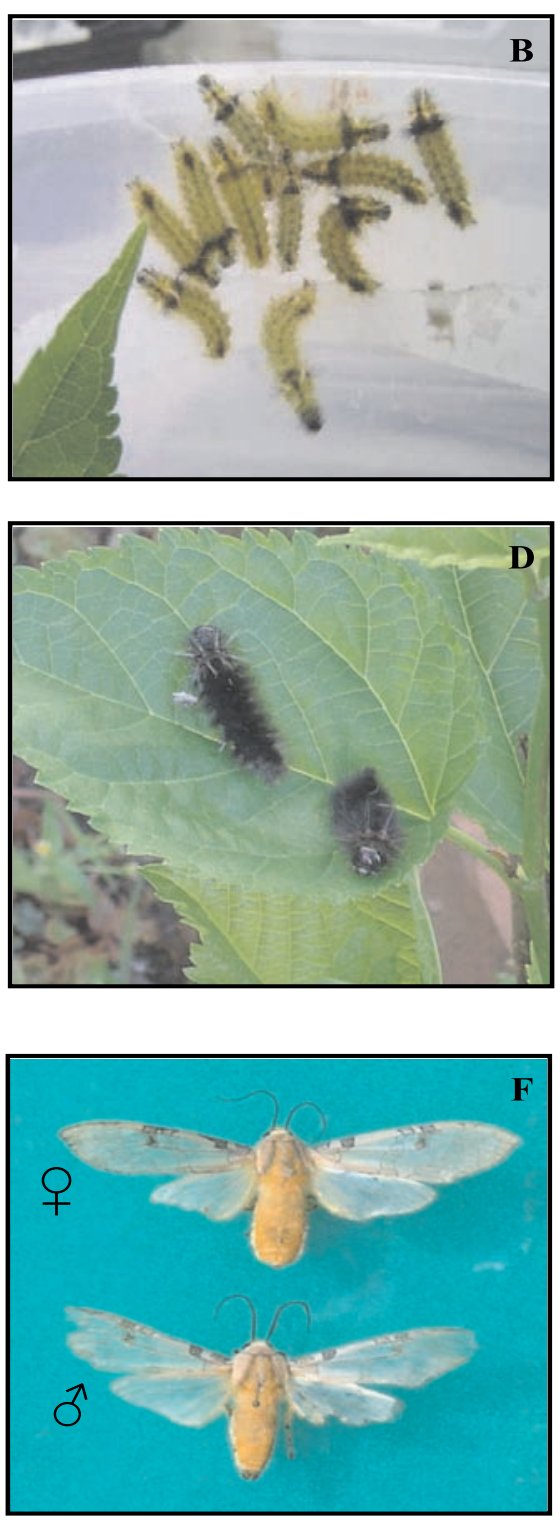

Figura 1 - Ovos (A), lagartas de terceiro estádio (B), lagartas de sexto estádio (C), lagartas de sexto estádio próximas da fase de pré-pupa (D), pupas com ou sem casulo (E) e adultos de Halysidota pearsoni (Lepidoptera: Arctiidae).

Figure 1 - Eggs (A), third instar caterpillars (B), sixth instar caterpillars $(C)$, sixth instar caterpillars near the pre-pupa stage (D), pupae with or without cocoon (E), and adults of Halysidota pearsoni (Lepidoptera: Arctiidae). 


\subsection{Larvas}

A duração (dias) e a viabilidade larval (\%) de $H$. pearsoni foram de 28,00 e 91,78 $\pm 3,24$, respectivamente, com seis estádios para essa espécie alimentando-se de folhas de M. alba (Figura 2). Lagartas de H. pearsoni apresentam comportamento gregário até o terceiro estádio, quando medem, aproximadamente, 14,1 mm de comprimento e 3,7 mm de largura, cabeça com 1,89 mm de largura e coloração marrom escura e corpo coberto por pêlos verde-claros. Apresentam manchas escuras com pêlos longos esbranquiçados no tórax e no extremo do abdome (Figura 1B); a região ventral do corpo é marrom-amarelada. Lagartas de quarto, quinto e sexto estádios não têm comportamento gregário, mas permanecem próximas umas das outras, alimentando-se de folhas de $M$. alba. Nesse último estádio, as lagartas medem $43,00 \mathrm{~mm}$ de comprimento e $6,50 \mathrm{~mm}$ de largura; cabeça com 4,53 mm, de coloração marromescura, corpo coberto de pêlos escuros com mancha vermelha alaranjada e pêlos longos esbranquiçados no tórax e extremo do abdome (Figura 1C). Outra espécie desse gênero, $H$. orientalis, teve suas lagartas relatadas em Piracicaba, São Paulo, alimentando-se de folhas de M. alba. As lagartas dessa espécie medem $45 \mathrm{~mm}$ de comprimento e $7 \mathrm{~mm}$ de largura no último estádio (SOTOSANCHEZ et al., 2004) e apresentam coloração e características morfológicas semelhantes às de $H$. pearsoni. $H$. interlineata também foi relatada em folhas de $M$. alba, porém no Estado do Rio de Janeiro (SILVA et al., 1968).

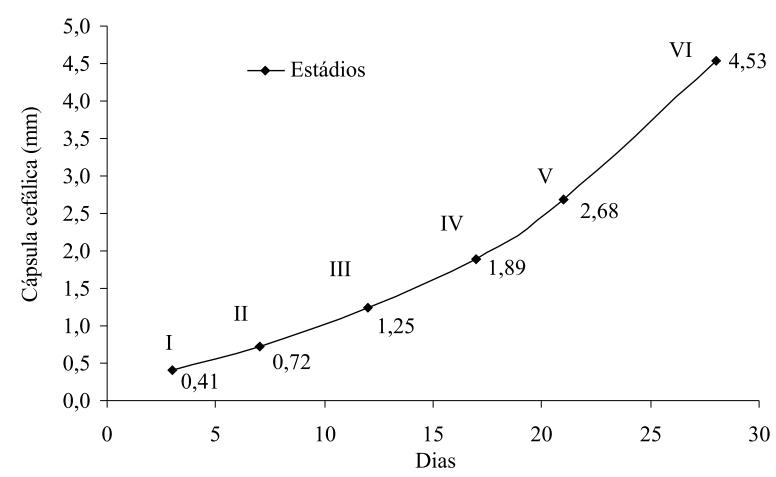

Figura 2 - Largura da cápsula cefálica e duração dos estádios de Halysidota pearsoni (Lepidoptera: Arctiidae) com folhas de Morus alba a $25 \pm 2{ }^{\circ} \mathrm{C}, 70 \pm 10 \%$ de umidade relativa e fotofase de 12 horas.

Figure 2 - Width of the head capsule and duration of the instars of Halysidota pearsoni (Lepidoptera: Arctiidae) fed with leaves of Morus alba at 25 $\pm 2{ }^{\circ} \mathrm{C}, 70 \pm 10 \%$ relative humidity and photo phase of 12 hours.

R. Árvore, Viçosa-MG, v.31, n.1, p.157-161, 2007

\subsection{Pupas}

A duração (dias) e a viabilidade (\%) da fase de pré-pupa e pupa de $H$. pearsoni foram de 7,00 $\pm 0,00$; $70,15 \pm 5,63$ e 19,39 $\pm 0,74 ; \mathrm{e} 93,62 \pm 3,60$, respectivamente, com folhas de $M$. alba. O peso da pupa (mg) desse inseto foi de 464,16 \pm 7,70. A lagarta de $H$. pearsoni diminui seus movimentos e perde parte de seus pêlos ao iniciar a fase de pré-pupa (Figura 1D). As pupas desse lepidóptero são marrom-escuras, com aproximadamente $18 \mathrm{~mm}$ de comprimento e 7,3 $\mathrm{mm}$ de largura, com casulo oval e de coloração escuroacinzentada, com cerca de $20,0 \mathrm{~mm}$ de comprimento e $8,5 \mathrm{~mm}$ de largura (Figura 1E). Os palpos labiais de H. pearsoni são visíveis, atingindo aproximadamente a sexta parte do comprimento das maxilas. Isso é importante, pois a exposição dos palpos labiais e seu comprimento em relação às maxilas são características que permitem diferenciar as pupas de Halysidota daquelas de outros gêneros da família Arctiidae (MOSHER, 1969). Pupas de H. orientalis são marromescuras, com aproximadamente $19 \mathrm{~mm}$ de comprimento e 7,6 mm de largura. Apresentam mesonoto convexo, concavidade na altura do metanoto e segmentos abdominais moderadamente achatados, as tecas alares anteriores, antenas e palpos maxilares terminam sobre o quarto segmento abdominal e as tíbias e tarsos das pernas protorácicas e mesotorácicas são evidentes (SOTO-SANCHEZ et al., 2004).

\subsection{Adultos}

A longevidade (dias) de machos e fêmeas de $H$. pearsoni foi de 7,40 $\pm 0,34 \mathrm{e} 9,50 \pm 0,45$, respectivamente, com folhas de $M$. alba. Machos e fêmeas desse inseto apresentam envergadura de asas de 27 a 31 e de 30 a $33 \mathrm{~mm}$, respectivamente, com a parte dorsal das asas de coloração bege com manchas pretas, abdome com coloração amarelo-alaranjada, antenas pectinadas, hábito noturno e, quando em repouso, permanecem com as asas fechadas em formato de telhado. O dimorfismo sexual de $H$. pearsoni é pouco acentuado, mas pode ser observado no formato da extremidade abdominal, que é mais afilado no macho (Figura 3F). Outras espécies dessa família como Eupseudosoma aberrans Schaus, 1905 (Lepidoptera: Arctiidae) apresentam adultos bastante parecidos, mas as antenas dos machos são serrilhadas e as das fêmeas, filiformes (ZANUNCIO, 1993). Espécies do gênero Halysidota são semelhantes e, por isso, para diferenciá-las há necessidade de se comparar, 
além do padrão alar, a genitália do macho (WATSON, 1980). Pesquisas relacionadas à descrição de genomas de várias famílias de lepidópteros têm sido realizadas e poderão facilitar a identificação precisa de espécies da família Arctiidae (GREGORY e HEBERT, 2003).

Adultos de Halysidota sp. foram coletados em plantio de Eucalyptus sp. em Minas Gerais (ZANUNCIO et al., 1989) e em fragmentos de floresta estacional residual em Itaara, Rio Grande do Sul (VIANA e COSTA, 2001), indicando que esse lepidóptero também está presente em plantios de eucalipto em várias regiões do Brasil. Desse modo, é importante definir os hospedeiros de $H$. pearsoni nas áreas reflorestadas com eucalipto e verificar se essa espécie pode se desenvolver neles. Essas informações poderão ser utilizadas no manejo integrado de $H$. pearsoni, caso essa espécie cause dano econômico.

\section{CONCLUSÕES}

H. pearsoni concluiu seu ciclo biológico, em condições de laboratório, alimentando-se de folhas de amoreira.

A elevada capacidade de reprodução de $H$. pearsoni indica que esse lepidóptero tem potencial para alcançar "status" de praga em plantios de amoreira.

\section{AGRADECIMENTOS}

Ao Conselho Nacional de Desenvolvimento Científico e Tecnológico (CNPq), à Coordenação de Aperfeiçoamento de Pessoal de Nível Superior (CAPES) e à Fundação de Amparo à Pesquisa do Estado de Minas Gerais (FAPEMIG), pelo apoio financeiro.

\section{REFERÊNCIAS}

BENTANCOURT, C.M.; SCATONI, Y.B. Biologia de Mallocephala deserticola Berg (Lepidoptera: Arctiidae). Anais da Sociedade Entomológica do Brasil, v.27, n.2, p.213-221, 1998.

FONSECA, A.S.; FONSECA, T.C. A cultura da amoreira e criação do bicho da seda: Sericicultura. São Paulo: Nobel, 1986. 246p.

GALLO, D. et al. Entomologia Agrícola. Piracicaba: FEALQ, 2002.920p.

GREGORY, T.R.; HEBERT, P.D.N. Genome size variation in lepidopteran insects. Canadian Journal of Zoology, v.81, n.1, p.1399-1405, 2003.
MOSHER, E. Lepidoptera pupae: Five collected works on the pupae of North American Lepidoptera. Michigan: Entomological Reprint Specialists, 1969. 323p.

SANTOS, G. P. et al. Bionomia e morfologia de Idalus admirabilis (Lepidoptera: Arctiidae) alimentada com folhas de Eucalyptus urophylla. Revista Árvore, v.30, n.1, p.123-127, 2006.

SILVA, A.G.A. et al. Quarto catálogo dos insetos que vivem nas plantas do Brasil. Seus parasitos e predadores. Insetos, hospedeiros e inimigos naturais. Rio de Janeiro: Ministério da Agricultura, 1968. Parte 2. Tomo 1.622p.

SOTO-SANCHEZ, S.; ROMANO, F.C.B.; NAKANO, O. Ocorrência de Halysidota orientalis Rothschild (Lepidoptera: Arctiidae) em amoreira (Morus alba L.) no estado de São Paulo. Neotropical Entomology, v.33, n.4, p.517-518, 2004.

VIANA, T.M.B.; COSTA, E.C. Lepidópteros associados a duas comunidades florestais em Itaara, RS. Ciência Florestal, v.11, n.1, p.67-80, 2001.

WATSON, A. A revision of the Halysidota tessellaris species-group (Halysidota sensu stricto) (Lepidoptera: Arctiidae). Bulletin of the British Museum (Natural History). Entomology, v.40, n.1, p.36-38, 1980.

WATSON, A.; GOODGER, D.T. Catalogue of the Neotropical Tiger-moths. Bulletin of the British Museum Natural History, v.1, n.1, p.1-71, 1986.

ZANUNCIO, J.C. Lepidópteros desfolhadores de eucalipto: Biologia, Ecologia e controle. Viçosa, MG: Instituto de Pesquisas e Estudos Florestais e Sociedade de Investigações Florestais, 1993. 140p.

ZANUNCIO, J.C. et al. Levantamento e flutuação populacional de lepidópteros associados à eucaliptocultura: 3-Região do Alto São Francisco, Minas Gerais, março de 1998 a fevereiro de 1989. IPEF, v.41/42, n.1, p.77-82, 1989.

R. Árvore, Viçosa-MG, v.31, n.1, p.157-161, 2007 
